\title{
Enhancement of the stability of the flow focusing technique for low-viscosity liquids
}

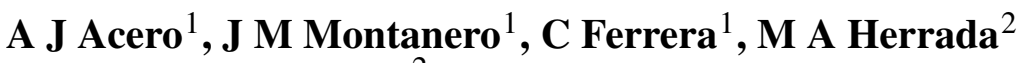 \\ and A M Gañán-Calvo ${ }^{2}$ \\ ${ }^{1}$ Department of Mechanical, Energetic and Material Engineering, University of Extremadura, \\ Avda. de Elvas s/n, E-06006 Badajoz, Spain \\ ${ }^{2}$ Department of Aerospace Engineering and Fluid Mechanics, University of Seville, \\ Camino de los Descubrimientos s/n, E-41092 Sevilla, Spain \\ E-mail: jmm@unex.es
}

Received 28 March 2012, in final form 7 September 2012

Published 19 October 2012

Online at stacks.iop.org/JMM/22/115039

\begin{abstract}
We propose a modified flow focusing configuration to produce low-viscosity microjets at much smaller flow rates than those reached by the standard configuration. In the modified flow focusing device, a sharpened rod blocks the recirculation cell appearing in the tapering liquid meniscus for low flow rates, which considerably improves its stability. We measured the minimum flow rates attainable with the modified configuration and compared the results with the corresponding values for the standard technique. For moderate and large applied pressure drops, the minimum flow rate reached with the modified configuration was about five times smaller than its counterpart in the standard configuration. The Weber numbers of the jets produced with the modified flow focusing configuration were considerably smaller than those with the standard technique. Numerical simulations were conducted to show how the presence of the inner rod substantially changes the flow pattern in the liquid meniscus.
\end{abstract}

\section{Introduction}

The controlled production of very small fluid entities such as drops, bubbles, emulsions and capsules has been extensively investigated over the last two decades because of its enormous relevance for a great variety of applications. Surfactants [1], electrical forces [2] or thermal gradients [3] can be used to produce fluid particles of varied morphology of micrometer or nanometer size. Extensional co-flows constitute a remarkable class of techniques in which viscous $[4,5]$ and/or pressure [6] forces stretch an interface until a small jet is emitted. The gas-liquid flow focusing technique working in the steady conejet mode uses the pressure gradient induced by an outer gas stream to 'focus' a steady liquid meniscus from whose tip a microjet is emitted. Both the liquid microjet and the coflowing gas stream cross a discharge orifice whose diameter is much larger than that of the microjet. Gas-liquid flow focusing allows the mass production of ultrafine and monodisperse sprays, microcapsules and microparticles in a highly controlled manner.
The control achieved in the flow focusing technique fundamentally stems from the delicate balance between liquid inertia and surface tension forces appearing for Weber numbers below a few tens [6], which guarantees that the breakup of the issuing microjet is essentially caused by varicose free-surface deformations. The steady cone-jet mode is less stable as the size of the feeding capillary and/or discharge orifice increases. Specifically, for a given pressure drop applied to the gas stream, the minimum flow rate (and thus the Weber number of the emitted jet) for which the steady cone-jet mode can be obtained increases as the size of the device increases. Therefore, a certain compromise must be reached when selecting the size of the flow focusing atomizer: relatively large devices are easier to manufacture and have higher productivity, whilst smaller configurations can attain lower Weber numbers, which improves the outcome quality. One is faced with a serious design problem (e.g., in multiplexed devices consisting of a number of flow focusing tips) to meet the high productivity and quality conditions demanded in many applications. Any modification of the standard flow focusing configuration which 


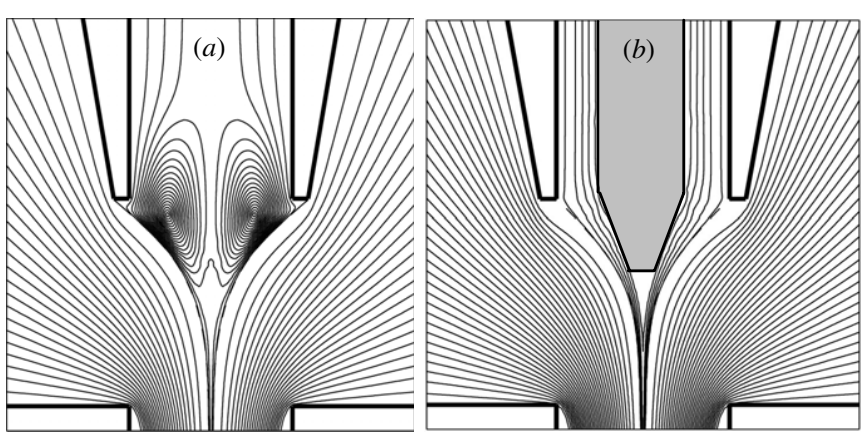

Figure 1. The standard $(a)$ and modified $(b)$ flow focusing configurations.

enhances the stability of the cone-jet mode would have obvious technological implications.

Studies of the mechanisms by which the cone-jet mode destabilizes at the minimum flow rate [7-11] show that many of the experimental results obtained for low/moderate viscosity liquids cannot be explained by the well-known convective/absolute instability transition [12] of the issuing liquid jet. This occurs not only in the classical plate-orifice configuration, but also when the orifice is replaced by a converging-diverging nozzle [13, 14]. In fact, the use of converging-diverging nozzles to produce the focusing effect entails certain advantages over the classical plate-orifice configuration, but does not stabilize the meniscus in the limit of small flow rates.

The appearance of a conspicuous recirculation cell inside the tapering liquid meniscus has been unequivocally linked to the stability limit of the steady cone-jet mode for low-viscosity liquids [7, 8, 10, 11]. Thus, an immediate proposition to extend the stability limit of flow focusing is the use of some means to destroy that recirculation cell. In electrospray studies (see, e.g., [15]), the system stability was substantially enhanced by the use of a sharpened rod protruding from the mouth of the feeding capillary. In this case, the stability enhancement may be attributable to a possible charge injection mechanism at the rod tip, not necessarily to the obliteration of internal recirculation motions [16]. One can legitimately inquire whether a similar configuration would also be beneficial in flow focusing.

Figure 1 shows the flow pattern of a low-viscosity liquid in the standard flow focusing device (a) and the modified configuration (b) proposed in this work. In the first case, the liquid flows close to the meniscus free surface toward its tip dragged by the tangential viscous stresses exerted by the outer gas stream. The pressure increases in the meniscus tip because momentum accumulates in that region. This increase of pressure pumps the liquid back through the meniscus bulk, which gives rise to a recirculation pattern for small enough injected flow rates. Numerical simulations [7] and experiments $[10,11]$ have shown that, for sufficiently large gas pressures, there is a critical flow rate below which this flow pattern becomes unstable. In this case, the jet emission halts, and the meniscus inflates and deflates, intermittently ejecting liquid ligaments.
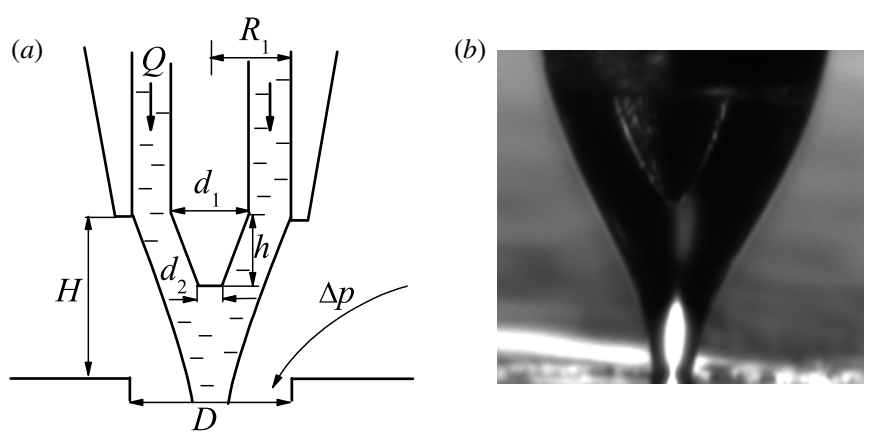

Figure 2. (a) Sketch of the modified flow focusing configuration. (b) Image of a liquid meniscus formed in the course of the experiments. To better appreciate the inner rod, the image was acquired for $H$ and $Q$ values much larger than those considered in our analysis.

The liquid motion is substantially changed by introducing a sharpened rod in the modified configuration. This rod 'arranges' the streamlines and 'directs' the flow in the meniscus tip, which prevents large recirculation cells from being formed even for very small injected flow rates and very large applied pressure drops. In this case, a new mechanism (probably associated with the surface tension) is responsible for the flow instability. In this work, the minimum flow rates attainable with the modified configuration were measured and compared with the corresponding values for the standard technique. We shall show that the use of an internal rod to destroy recirculation motions in the liquid meniscus reduces by up to five times the minimum flow rate of the standard flow focusing configuration.

\section{Methods}

\subsection{Experimental method}

Figure 2 shows the fluid configuration considered in our experiments. An axisymmetric meniscus is formed by injecting a liquid at a constant flow rate $Q$ through a feeding capillary of inner radius $R_{1}=100 \mu \mathrm{m}$. A steel calibration rod of diameter $d_{1}=100 \mu \mathrm{m}$ was positioned concentrically inside the feeding capillary. The rod end was previously sharpened to give it a conical shape characterized by the lengths $h=$ $110 \mu \mathrm{m}$ and $d_{2}=15 \mu \mathrm{m}$. The feeding capillary was located at a distance $H$ from a plate with an orifice of diameter $D=$ $200 \mu \mathrm{m}$. The energy necessary to form and expel the jet comes from the pressure drop $\Delta p$ applied to an air stream which co-flows with the liquid jet across the plate orifice.

The control parameters of the experiments are the liquid flow rate $Q$, the applied pressure drop $\Delta p$ and the capillary-toorifice distance $H$. Naturally, the results can also be affected by the liquid density $\rho$ and viscosity $\mu$, as well as by the surface tension $\sigma$. In order to study the influence of the liquid properties on the flow stability, we considered two low-viscosity liquids with different viscosities and surface tensions: distilled water $\left(\rho=998 \mathrm{~kg} \mathrm{~m}^{-3}, \mu=1 \times 10^{-3}\right.$ $\left.\mathrm{kg}(\mathrm{ms})^{-1}, \sigma=0.072 \mathrm{Nm}^{-1}\right)$ and hexadecane $(\rho=$ $\left.773 \mathrm{~kg} \mathrm{~m}^{-3}, \mu=3.03 \times 10^{-3} \mathrm{~kg}(\mathrm{~ms})^{-1}, \sigma=0.026 \mathrm{~N} \mathrm{~m}^{-1}\right)$. 


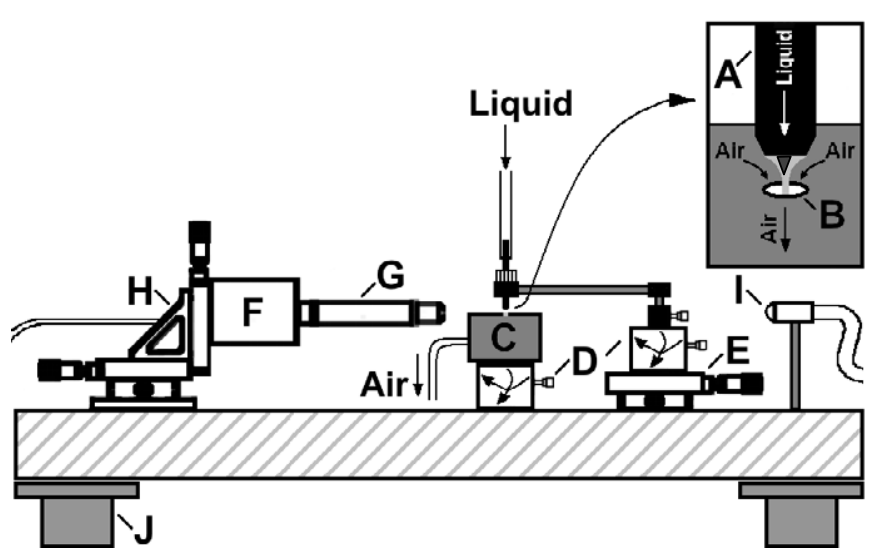

Figure 3. Experimental setup: (A) capillary, (B) plate with orifice, (C) suction cell, (D) orientation systems, (E) translation stage, (F) high-speed video camera, $(\mathrm{G})$ optical lenses, $(\mathrm{H})$ triaxial translation stage, (I) optical fiber and (J) anti-vibration isolation system.

The fluid configuration analyzed in this work is the same as that studied in the earlier work [10] except for the presence of the inner rod.

Figure 3 shows the experimental setup used to determine the liquid meniscus behavior. The liquid was injected by a syringe pump (HARVARD APPARATUS PHD 4400) powered by a stepper motor through a capillary (A) located in front of a plate with an orifice (B). The plate covered the upper face of a cubic cell (C) in which a prescribed negative gauge pressure was applied by using a suction pump. We used two high-precision orientation systems (D) and a translation stage (E) to ensure the correct alignment of the flow focusing elements, and to set the capillary-to-orifice distance $H$. The liquid meniscus was formed in the open air due to the action of the air stream suctioned by the cell through the plate orifice.

Digital images of the liquid meniscus consisting of $360 \times 200$ pixels were acquired at 1800 frames per second with an exposure time of $30 \mu$ s using a CMOS camera (PнотоNFocus MV-D1024-160) (F). The camera was equipped with optical lenses (a Mitutoyo 10× magnification zoomobjective and an OPTEM 70XL set of lenses with variable magnification from $0.75 \times$ to $5.25 \times)(\mathrm{G})$ providing a frame covering an area of about $533 \times 296 \mu \mathrm{m}$. The magnification obtained was approximately $1.48 \mu \mathrm{m} /$ pixel. The camera could be displaced both horizontally and vertically using a triaxial translation stage $(\mathrm{H})$ to focus the liquid meniscus. The fluid configuration was illuminated from the back side by cool white light provided by an optical fiber (I) connected to a light source. We also acquired images of the liquid meniscus by using an auxiliary CCD camera (not shown in figure 3) with an optical axis perpendicular to that of the CMOS camera. The use of the two cameras allowed us to check that flow focusing was axisymmetric and that no contact between the meniscus free surface and the inner rod took place. All these elements were mounted on an optical table with a pneumatic anti-vibration isolation system $(\mathbf{J})$ to damp the vibrations coming from the building.

The experimental procedure consisted of the following steps. Firstly, the capillary-to-orifice distance $H$ was fixed.

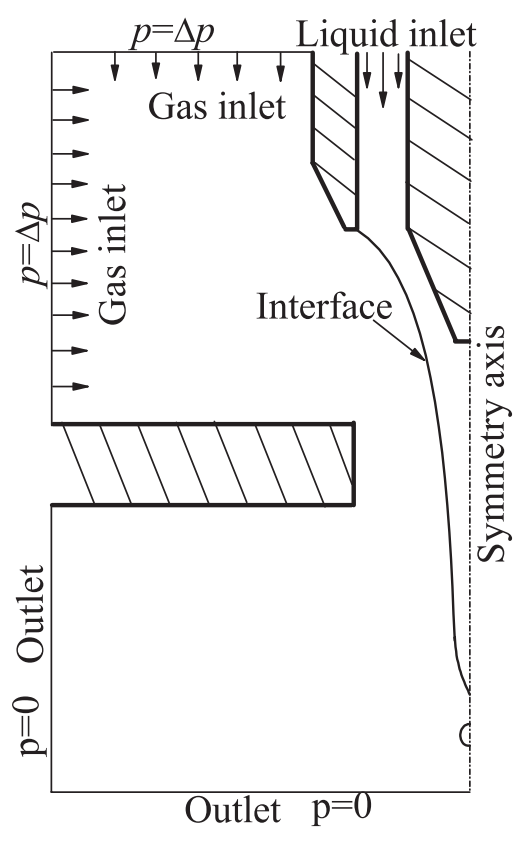

Figure 4. Boundary conditions in the numerical simulations.

Secondly, a constant pressure drop $\Delta p$ was applied to the air stream. Thirdly, the liquid was injected at a flow rate $Q$ sufficiently high to ensure steady jetting stability. Lastly, the flow rate was reduced in steps of $0.1 \mathrm{ml} \mathrm{h}^{-1}$ until the steady jetting became unstable. We repeated the same experimental procedure for different values of the applied pressure drop $\Delta p$ and the capillary-to-orifice distance $H$. Images of the liquid meniscus at the minimum flow rate were recorded to measure the meniscus radius $R_{j}$ just in front of the plate orifice.

\subsection{Numerical method}

In order to determine the influence of the inner rod on the flow topology, the streamlines were calculated with a finite volume scheme. This scheme was provided by the commercial solver FLUENT v 6.3 (laminar unsteady) to resolve the mass continuity, momentum conservation and liquid volume fraction equations in the incompressible regime. The geometrical configurations analyzed were the same as those considered in the experiments. The radius of the outer boundary of the simulated fluid domain was $1 \mathrm{~mm}$. The mesh was sufficiently refined to capture the strong velocity gradients experienced by the gas flow in the orifice region and to avoid numerical diffusion of the interface.

Figure 4 shows the boundary conditions used in the simulations. The velocity profile corresponding to fully developed flow with a flow rate $Q$ was prescribed at the liquid inlet. At the gas inlet, the gauge pressure $\Delta p$ was imposed, and the velocity direction was set normal to the boundary. Zero gauge pressure was prescribed at the outlet, which was located far from the jet breakup region. The nonslip boundary condition was imposed at the solid walls. The simulations started from rest by injecting a liquid flow rate $Q$ sufficiently high to obtain steady jetting. Once the steady jetting regime was reached, $Q$ was reduced progressively until it took the prescribed value. More details of the procedure can be found elsewhere [7]. 


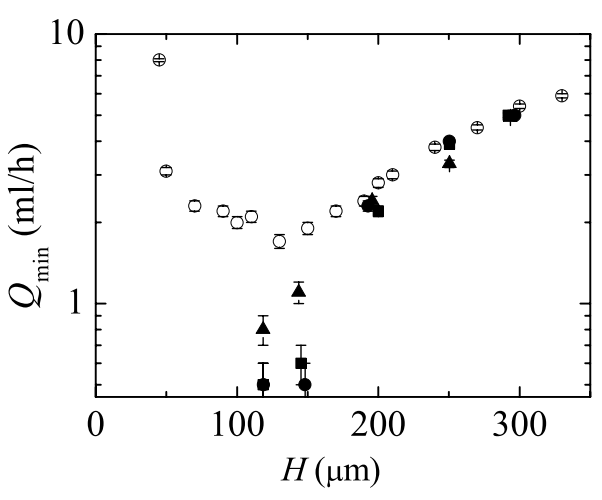

Figure 5. Minimum flow rate $Q \min$ of water as a function of the capillary-to-orifice distance $H$ for the standard (open symbols) and modified (solid symbols) flow focusing configurations. The triangles, squares and circles correspond to $\Delta p=100,150$ and 250 mbar, respectively.

\section{Results and discussion}

Figure 5 shows the minimum flow rate $Q_{\min }$ for a given $\Delta p$ value as a function of the capillary-to-orifice distance $H$. The figure also shows as a reference the results obtained for the standard flow focusing configuration [10]. As can be observed, the minimum flow rates for the standard and modified flow focusing devices approximately coincide for large $H$ values, which means that the inner rod plays no significant stabilizing role in that limit. This result was to be expected because the inner rod occupies a small portion of the meniscus volume in this case, and thus does not alter considerably the meniscus flow pattern. In contrast, the minimum flow rate for small $H$ $(H \gtrsim h, D / 2)$ is greatly affected by the presence of the rod. In the standard configuration, $Q_{\min }$ reaches a limiting value for $H \simeq D / 2$ [10]. The presence of the inner rod reduces this limiting value by almost five times. The stability of the modified flow focusing configuration was not explored for $H \lesssim h$ because in this case the inner rod enters the plate orifice and one is unable to observe the behavior of the entire meniscus. Hereafter, all the experimental results correspond to the capillary-to-orifice distance $H$ which approximately led to a limiting flow rate $(H=110$ and $140 \mu \mathrm{m}$ for the standard and modified configurations, respectively).

Figure 6 shows $Q_{\min }$ as a function of $\Delta p$ for water and hexadecane. The figure also shows as a reference the results obtained for the standard flow focusing configuration. As can be observed, the minimum flow rate was considerably reduced over a large range of $\Delta p$ by introducing the inner rod. Once the recirculation cell has been suppressed (or, at least, diminished) with the introduction of the inner rod, meniscus instability is probably caused by the surface tension retraction force, which partially explains the difference between the minimum flow rates of water and hexadecane in the modified flow focusing configuration.

Figure 7 shows the values of the critical Reynolds and Weber numbers

$$
\mathrm{Re}=\frac{\rho Q_{\min }}{\pi R_{j} \mu}, \quad \mathrm{We}=\frac{\rho Q_{\mathrm{min}}^{2}}{\pi^{2} R_{j}^{3} \sigma},
$$

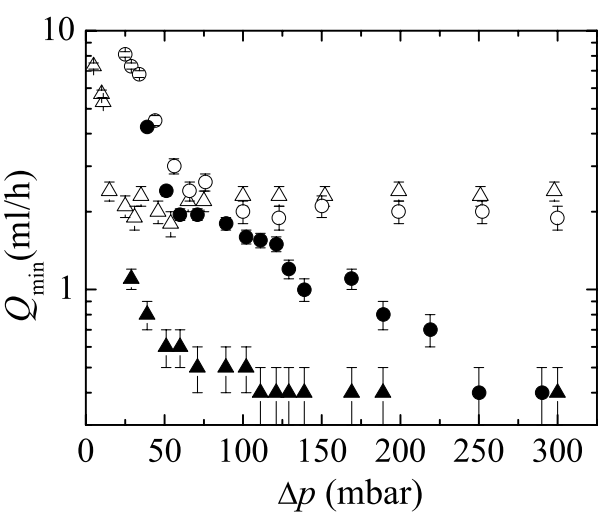

Figure 6. Minimum flow rate $Q_{\text {min }}$ as a function of the applied pressure drop $\Delta p$ for the standard (open symbols) and modified (solid symbols) flow focusing configurations. The circles and triangles correspond to water and hexadecane, respectively.

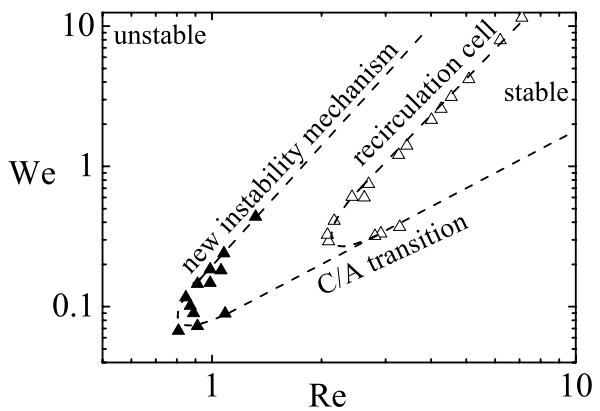

Figure 7. Critical values of the Reynolds and Weber numbers obtained for hexadecane with the standard (open symbols) and modified (solid symbols) flow focusing configurations. The dashed lines are guides to the eye.

for hexadecane. We also plot in the figure the corresponding values of the standard flow focusing configuration. One can distinguish two types of stability limits. The right-hand dashed line, common to the two sets of data, corresponds to low applied pressure drops. In this case, the instability is caused by the convective/absolute instability transition [12] taking place in the emitted jet [10]. This instability mechanism is not significantly affected by the presence of the inner rod. The Weber numbers for which the convective/absolute instability transition takes place deviate significantly from the Leib and Goldstein prediction [17] because the jet's velocity profile is not uniform. Owing to the co-flowing gas stream, the jet's free surface moves with a velocity greater than that of the bulk, which naturally favors the jet to sweep growing surface waves downstream, displacing the onset of dripping to lower flow rates (Weber numbers) [18]. The left-hand dashed lines indicate the instability limits for moderate/large pressure drops. In the standard configuration, the recirculation cell appearing in the liquid meniscus for low flow rates becomes unstable. The sharpened rod introduced in the modified configuration arranges the streamlines shifting the stability limit to much lower values of the flow rate (i.e. Reynolds and Weber numbers). In this case, a new mechanism (probably associated with the surface tension) is responsible for the flow instability. Overall, considerably smaller values of the Weber 

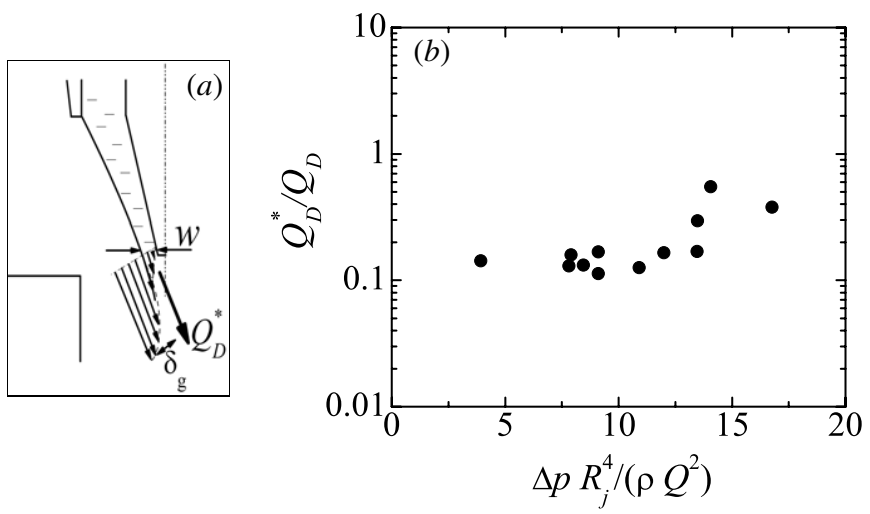

Figure 8. (a) Sketch to show the meaning of the quantities used to estimate $Q_{D}^{*}$. (b) $Q_{D}^{*} / Q_{D}$ as a function of the dimensionless pressure $\Delta p R_{j}^{4} /\left(\rho Q^{2}\right)$ for the minimum flow rates obtained with hexadecane.

number could be reached with the modified flow focusing configuration, which constitutes an important advantage in some droplet production applications.

For not very low-viscosity liquids (e.g., hexadecane), one can easily estimate the flow rate that the air stream sets in motion by dragging the liquid layer next to the free surface. To this end, we shall assume that the free surface velocity $U_{s}$ is much smaller than the velocity $U_{g} \sim\left(\Delta p / \rho_{g}\right)^{1 / 2}\left(\rho_{g}\right.$ is the gas density) of the gas stream (see figure $8(a)$ ). In this case, the integral form of the momentum equation for the accelerated gas boundary layer leads to $\rho_{g} U_{g}^{2} H^{-1} \sim \mu_{g} U_{g} / \delta_{g}^{2}$, where $\mu_{g}$ is the gas viscosity, and $\delta_{g}$ is the characteristic boundary layer thickness. The balance of momentum on the two sides of the meniscus free surface yields $\mu U_{s} / w \sim \mu_{g} U_{g} / \delta_{g}$, where $w$ is the thickness of the Couette stream formed next to the tip of the inner rod. This thickness can be measured from the meniscus images. From the above two equations, one can estimate the free surface velocity, $U_{s} \sim w \mu^{-1}\left(\rho_{g} \mu_{g} U_{g}^{3} H^{-1}\right)^{1 / 2}$, and the dragged flow rate, $d_{2} w U_{s}$. In this way, one obtains the characteristic flow rate

$$
Q_{D}^{*} \equiv \frac{d_{2} w^{2}}{\mu}\left(\rho_{g}^{-1 / 2} \mu_{g} \Delta p^{3 / 2} H^{-1}\right)^{1 / 2} .
$$

This quantity can be compared with its counterpart $Q_{D} \equiv D \mu / \rho$ for the standard flow focusing configuration $[7,8,11]$ (see figure $8(b)$ ). As can be observed, the dragged flow rate in the new configuration is significantly smaller than that of the standard one, which inhibits the appearance of recirculation cells. Interestingly, the average value of $Q_{D}^{*} / Q_{D}$ is 0.21 , similar to the ratio of minimum flow rates reached by the two configurations.

The inner rod is an energy sink which prevents large recirculation cells from appearing for flow rates at which that pattern arises in the standard configuration. To assess the energy efficiency of this stabilizing mechanism, the radius $R_{j}$ of the liquid meniscus measured just in front of the plate orifice was compared with the value

$$
R_{0}=\left(\frac{\rho Q^{2}}{2 \pi^{2} \Delta p}\right)^{1 / 4} .
$$

This reference value is obtained by assuming that the injected energy per unit volume, $\Delta p$, is entirely transformed into kinetic

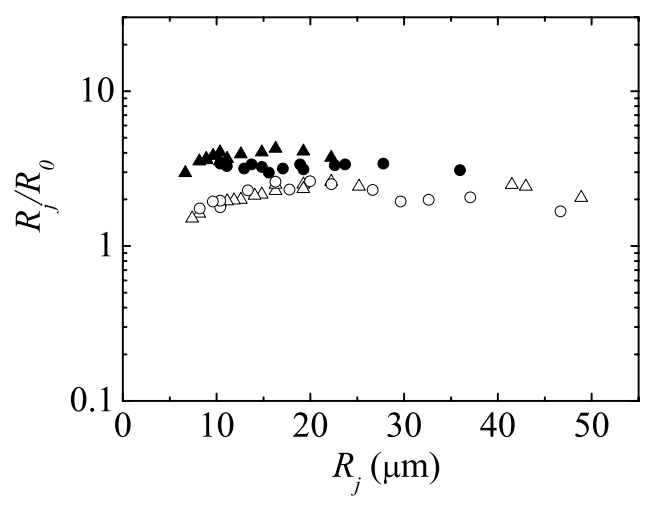

Figure 9. Radius $R_{j}$ of the liquid meniscus measured for the minimum flow rate $Q_{\min }$ just in front of the plate orifice with the standard (open symbols) and modified (solid symbols) flow focusing configurations. The circles and triangles correspond to water and hexadecane, respectively. $R_{0}$ is the radius calculated from (3).

energy. Figure 9 shows the results for both the standard and modified configurations. The ratio $R_{j} / R_{0}$ is greater than unity in all the cases because only a part of the injected energy is transferred to the liquid between the feeding capillary end and the plate orifice. In fact, the suction caused by the gas stream continues inside the orifice, where a major amount of energy is transferred to the liquid. Similar jet sizes were obtained with the two configurations. The values of the ratio $R_{j} / R_{0}$ measured in the modified configuration are greater than those in the standard device due to the energy dissipated by viscous friction with the inner rod. This effect is slightly more notable for hexadecane because it is more viscous than water.

The influence of the inner rod on the flow topology was determined from the finite volume scheme described in section 2.2. Figure 10 shows the streamlines calculated for $Q=$ $3 \mathrm{ml} \mathrm{h}^{-1}$ and $\Delta p=200 \mathrm{mbar}$. In this case, the steady jetting regime can be obtained with both the standard and modified flow focusing configurations (see figure 6). In the absence of the inner rod, two large recirculation cells appear in the liquid meniscus for both water and hexadecane. The introduction of the sharpened rod into the water meniscus greatly reduces the size of those cells, and makes them disappear in the hexadecane case. If the flow rate is lowered down to $Q=1.5 \mathrm{ml} \mathrm{h}^{-1}$ while keeping the applied pressure drop, the standard flow focusing configuration drips (see figure 6). In contrast, the change in the flow pattern produced by the inner rod stabilizes the meniscus in the modified technique. Figure 11 shows the streamlines obtained in this case for both liquids. Small recirculation cells were produced in the water film due to its low viscosity. In the hexadecane case, the streamlines were perfectly arranged by the inner rod, and the fluid particles were smoothly directed toward the film tip. The different behaviors exhibited by water and hexadecane in the modified configuration may partially explain the difference between the minimum flow rates reached in both cases.

To summarize, a novel flow focusing technique has been proposed and tested. This technique allowed us to steadily produce low-viscosity jets at flow rates much smaller than 


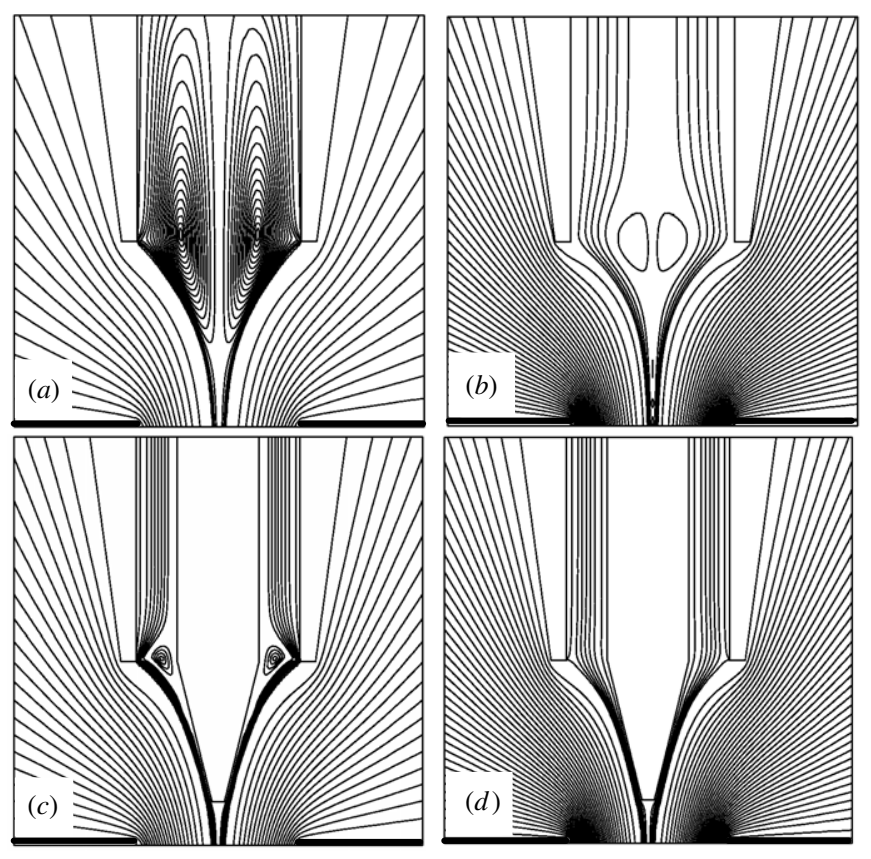

Figure 10. Streamlines calculated for $H=140 \mu \mathrm{m}, Q=3 \mathrm{ml} \mathrm{h}^{-1}$ and $\Delta p=200$ mbar without $((a)$ and $(b))$ and with $((c)$ and $(d))$ the inner rod. The graphs $(a)$ and $(c)$ show the results obtained for water, while $(b)$ and $(d)$ correspond to hexadecane.
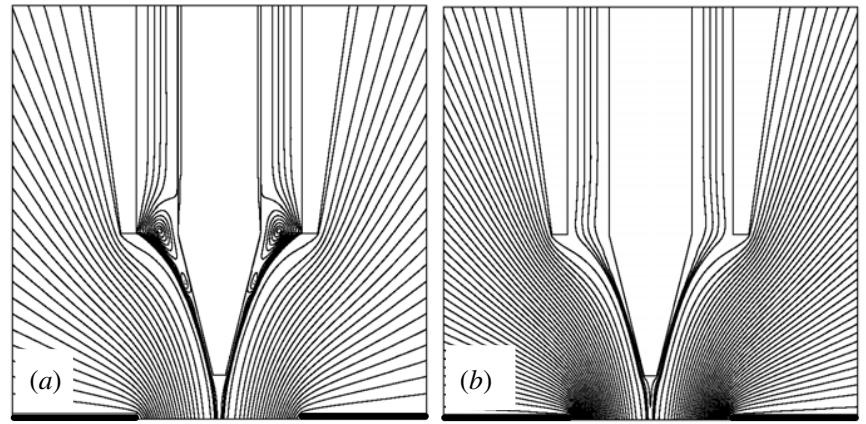

Figure 11. Streamlines calculated for $H=140 \mu \mathrm{m}, Q=1.5 \mathrm{ml} \mathrm{h}^{-1}$ and $\Delta p=200 \mathrm{mbar}$ with the inner rod. The graphs $(a)$ and $(b)$ show the results obtained for water and hexadecane, respectively.

those reached with the standard configuration [10]. The only new element of the modified configuration is a sharpened rod located inside the liquid meniscus to eliminate the large recirculation cell appearing close to the minimum flow rate stability limit. This recirculation cell is responsible for the loss of stability of low-viscosity menisci in that limit $[7,10,11]$.

The Weber numbers of the jets produced with the modified flow focusing configuration were considerably smaller than those reached with the standard technique. Therefore, one may expect that droplets with smaller kinetic/interfacial energy ratios can be obtained with the proposed configuration from the jet's breakage. This constitutes an important technological advantage in, for example, microfabrication techniques by droplet deposition on a solid substrate, where the quality of the deposition process strongly depends on the kinetic/interfacial energy ratio of the droplets.

\section{Acknowledgments}

Partial support from the Ministry of Science and Education, Junta de Extremadura and Junta de Andalucía (Spain) through grants Nos. DPI2010-21103, GR10047 and P08-TEP-04128, respectively, is gratefully acknowledged.

\section{References}

[1] Eggleton C D, Tsai T-M and Stebe K J 2001 Tip streaming from a drop in the presence of surfactants Phys. Rev. Lett. 87048302

[2] Fernández de la Mora J 2007 The fluid dynamics of Taylor cones Annu. Rev. Fluid Mech. 39 217-43

[3] Basaran O A 2002 Small-scale free surface flows with breakup: drop formation and emerging applications $A l C h E$ J. 48 1842-8

[4] Cohen I, Hui L, Hougland J L, Mrksich M and Nagel S R 2001 Using selective withdrawal to coat microparticles Science 292 265-7

[5] Blanchette F and Zhang W W 2009 Force balance at the transition from selective withdrawal to viscous entrainment Phys. Rev. Lett. 102144501

[6] Gañán-Calvo A M 1998 Generation of steady liquid microthreads and micron-sized monodisperse sprays in gas streams Phys. Rev. Lett. 80 285-8

[7] Herrada M A, Gañán-Calvo A M, Ojeda-Monge A, Bluth B and Riesco-Chueca P 2008 Liquid flow focused by a gas: jetting, dripping, and recirculation Phys. Rev. E 78036323

[8] Gañán-Calvo A M and Montanero J M 2009 Revision of capillary cone-jet physics: electrospray and flow focusing Phys. Rev. E 79066305

[9] Si T, Li F, Yin X-Y and Yin X-Z 2009 Modes in flow focusing and instability of coaxial liquid-gas jets J. Fluid Mech. $6291-23$

[10] Vega E J, Montanero J M, Herrada M A and Gañán-Calvo A M 2010 Global and local instability of flow focusing: the influence of the geometry Phys. Fluids 22064105

[11] Montanero J M, Rebollo-Muñoz N, Herrada M A and Gañán-Calvo A M 2011 Global stability of the focusing effect of fluid jet flows Phys. Rev. E 82036309

[12] Huerre P and Monkewitz P A 1990 Local and global instabilities in spatially developing flows Annu. Rev. Fluid Mech. 22 473-537

[13] Montanero J M, Gañán-Calvo A M, Acero A J and Vega E J 2010 Micrometer glass nozzles for flow focusing J. Micromech. Microeng. 20075035

[14] Acero A J, Ferrera C, Montanero J M and Gañán-Calvo A M 2012 Focusing liquid microjets with nozzles $J$. Micromech. Microeng. 22065011

[15] Sen A K, Darabi J, Knapp D R and Liu J 2006 Modeling and characterization of a carbon fiber emitter for electrospray ionization J. Micromech. Microeng. 16 620-30

[16] Barrero A, Gañán-Calvo A M, Dávila J, Palacio A and Gómez-González E 1998 Low and high Reynolds number flows inside taylor cones Phys. Rev. E 58 7309-14

[17] Leib S J and Goldstein M E 1986 Convective and absolute instability of a viscous liquid jet Phys. Fluids 29 952-4

[18] Gañán-Calvo A M 2008 Unconditional jetting Phys. Rev. E 78026304 